\title{
Abstracts and Commentaries on Key Articles in the Literature
}

\author{
Reviewed by Wadie I. Najm, MD, MEd
}

\section{Acupuncture in the treatment of upper-limb lymphedema: results of a pilot study.}

Cassileth BR, Van Zee KJ, Yeung KS, et al. Cancer. 2013 Jul 1;119(13):2455-2461.

$\mathbf{R}$ ESEARCHERS From Memorial Sloan-Kettering Cancer Center, New York, New York, conducted this study. The study was supported in part by the Laurance S. Rockefeller Fund.

Lymphedema is a common consequence of breast-cancer surgery and is a major source of complications (infections, limited function, pain, etc.). This study aimed to evaluate the safety and potential efficacy of acupuncture for treating chronic upper-limb lymphedema.

Women were recruited for this pilot study if they were 18 years of age or older with unilateral lymphedema $(>2 \mathrm{~cm}$ in circumference difference between arms) and had a clinical diagnosis of lymphedema for at least 6 months and $<5$ years. Women previously treated with acupuncture for lymphedema; women using diuretics; or women with histories of metastatic cancer, autoimmune or fibroproliferative disorders, primary lymphedema, bone-marrow transplants; or women receiving current treatments with corticosteroids, myelosuppressive or stimulatory drugs were excluded from this study.

Enrolled subjects received acupuncture treatment twice weekly for 30 minutes over 4 consecutive weeks. Monthly follow-up calls were conducted for 6 months following completion of the treatment.

A total of 33 subjects (out of 37 who were enrolled) completed the study. Fourteen acupuncture points (TE 14, LI 15, LU 5, LI 4, ST 36, and SP 6 bilaterally; and CV12 and CV3) were needled by an experienced licensed acupuncturist. Acupuncture points were determined based on a literature review, practical experience, and the consensus of researchers.

Ipsilateral and contralateral arm circumference was measured at baseline and weekly before and after each treatment. Measurements were taken at two points: $10 \mathrm{~cm}$ above and $5 \mathrm{~cm}$ below the olecranon. The extent of breast cancer-related lymphedema (BCRL) was calculated by de- termining the difference in circumference of the affected and unaffected arms at the site with the largest baseline difference.

The median age of participants was 55. A median of 3.9 years elapsed from the patients' axillary surgery to the start of acupuncture. Most patients had been receiving standard lymphedema treatments before entering the study.

A total of 11 patients $(95 \%$ confidence interval [CI] $18 \%-$ $52 \%$ ) had a reduction of $\geq 30 \%$ in extent of BCRL after acupuncture treatment. Eighteen subjects (95\% CI, 36\%-72\%) experienced a reduction of $\geq 20 \%$. The mean reduction in the extent of BCRL was $0.90 \mathrm{~cm}(95 \% \mathrm{CI}, 0.72-1.07 \mathrm{~cm}$; $p<0.0005)$.

Sustained improvement for 4 months during the followup period occurred in 4 of 11 responders. Three additional responders reported sustained improvement for at least 4 weeks after treatment cessation. No serious adverse events were noted during the treatment and for the 6 months of follow-up interviews.

The researchers concluded that, overall, acupuncture provided by a qualified acupuncturist is safe and possibly effective for treating BCRL. The therapeutic and costreduction potential of acupuncture as a treatment for lymphedema may make this modality an important tool in the arsenal of lymphedema management. A randomized clinical trial is ongoing to confirm the results of this pilot study.

\section{Acupuncture for ovulation induction in polycystic ovary syndrome: a randomized controlled trial.}

Johansson J, Redman L, Veldhuis PP, et al. Am J Physiol Endocrinol Metab. 2013;304(9):E934-E943.

$\mathbf{T}$ HIS RANDOMIZED, CONTROLLED, AND BLINDED STUDY was conducted by researchers from various institutes, centers, and universities in Gothenburg, Sweden; Los Angeles, California; Charlottesville, Virginia; Quebec, Canada; Riyadh, Saudi Arabia; and Harbin, China. The study was financed by grants from the Swedish Research Council, 
the Jane and Dan Olsson Foundations, the Novo Nordisk Foundation, The Hjalmar Svensson Foundation, the Adlerbert Research Foundation, Wilhelm and Martina Lundgrens's Science Fund, the Swedish federal government under the LUA/ALF agreement, and the Regional Research and Development Agreement.

Researchers tested the hypothesis that frequent acupuncture treatment could improve ovulation frequency, luteinizing hormone (LH) secretion and pulsatility, and sex-steroid secretion in lean or overweight patients with polycystic ovary syndrome (PCOS).

The primary outcome measures were changes in $\mathrm{LH}$ pulsatility from baseline to the end of treatment and differences in ovulation frequency during the study.

Patients with PCOS were recruited by placing advertisements in local newspapers between June 2009 and September 2010. PCOS was diagnosed according to the Rotterdam criteria: ultrasound-verified (polycystic ovaries ( $\geq 12$ follicles of $2-9 \mathrm{~mm}$ and/or an ovarian volume $\geq 10 \mathrm{~mL}$ in one or both ovaries); oligo/amenorrhea; or clinical signs of hyperandrogenism (hirsutism or acne). Subjects were excluded if they were younger than 18 or older than 38 , had a body mass index $(\mathrm{BMI})>30$, had taken any pharmacologic treatments in the previous 3 months, or had breastfed or received acupuncture during the 24 weeks prior to inclusion in the study. Other exclusion criteria were cardiovascular disease, diabetes mellitus, and other endocrine disorders (such as congenital adrenal hyperplasia, Cushing's syndrome, or androgen-secreting tumors).

Thirty-two women with PCOS were equally randomized to receive either acupuncture with manual and lowfrequency electrical stimulation or to meetings with a physical therapist twice a week for 10-13 week. Randomization was computer-generated, and investigators were blinded until statistical analyses were performed. After randomization, each participant underwent a 10-13-week intervention period, after which the baseline measurements were repeated. Subjects in the acupuncture group were treated for 30 minutes, twice weekly for the 10-13 weeks, by two therapists. Use of two sets of 11 and 13 acupuncture points described in a previous study* was alternated every other treatment because of the intensity of the treatment.

Subjects in the control group met with physical therapists twice weekly for the 10-13 weeks to control for the attention involved in meeting with the therapists. Time was spent resting and listening to relaxing music, with the therapist entering the room every 10 minutes to ensure the patients' comfort and well-being in a manner that was similar to the acupuncture group.

Measurements were taken at baseline and were repeated within 1 week after the last treatment. Measurements were taken at menstrual cycle days 8-10 if ovulation had occurred. If no ovulation or bleeding had occurred during the study, measurements were taken on an arbitrary day of the cycle.
Anthropometrics included measurements of height, weight, waist and hip circumferences, sagittal diameter, and calculations of waist-to-hip ratio (WHR) and BMI $\left(\mathrm{kg} / \mathrm{m}^{2}\right)$. A foot-tofoot bioelectrical impedance system was used to measure the \% of body fat. The Ferriman-Gallwey (FG) score was used to assess hirsutism. Ovarian volume, endometrial thickness, and antral follicle $(<9 \mathrm{~mm})$ count were measured by ultrasound. Bleeding and progesterone measurements determined the number of ovulations per month that occurred during the study.

Twenty-eight women were included in the analyses- -12 in the attention control group and 16 in the acupuncture group. All participants were oligo/amenorrheic before the start of treatment, and the menstrual cycle pattern did not differ between the two groups.

During the study period, ovulation frequency was higher in the acupuncture group $(0.76 \pm 0.27$ versus $0.41 \pm 0.28$ ovulations per month; $p=0.002$ ). There were no changes in LH or cortisol pulsatility after treatment, except for an increase in $\mathrm{LH}$ within the acupuncture group. However, there were no between-group differences. There were no anthropometric differences between the groups. Significant changes in plasma glucose levels and Homeostasis Model Assessment scores from baseline to end of treatment occurred between groups, but no significant differences were seen within the groups. Acupuncture reduced circulating inhibin $B$ from baseline to end of treatment, but the between-group difference was not significant $(p=0.075)$. Circulating levels of estrone, estrone sulfate, estradiol, dehydroepiandrosterone, dehydroepiandrosterone sulfate, androstenedione, testosterone, free testosterone, dihydrotestosterone, androsterone glucuronide, androstane $3 \alpha, 17 \beta$ diol-3-glucuronide, and androstane-3 $\alpha, 17 \beta$-diol-17-glucuronide decreased within the acupuncture group and were significantly lower than in the control group for all of these except for androstenedione.

The researchers concluded that repeated acupuncture treatments with manual and electrical stimulation in lean/ overweight women with PCOS resulted in higher ovulation frequency during the treatment period than what occurred in the control group. These changes were not associated with a decrease in the LH pulsatility pattern. Instead, the effect on ovulation frequency appeared to be related mainly to a decrease in circulating sex steroids and their precursors. The reduction of both ovarian and adrenal serum sex steroids, with no concomitant effect on LH and cortisol pulsatility or secretion pattern, together with the within-group reduction of inhibin B levels, indicate that the effect of acupuncture is mainly of peripheral origin.

*Jedel E, Labrie F, Odén A, et al. Impact of electro-acupuncture and physical exercise on hyperandrogenism and oligo/amenorrhea in women with polycystic ovary syndrome: a randomized controlled trial. Am J Physiol Endocrinol Metab. 2011;300(1):E37E45. 


\section{Electrical stimulation of acupoint combinations against deep venous thrombosis in elderly bedridden patients after major surgery.}

Hou L, Chen C, Xu L, Yin P, Peng W. J Tradit Chin Med. 2013;33(2):187-193.

$\mathbf{R}$ ESEARCHERS FROM SEVERAL UNIVERSITIES AND HOSPITALS in Shanghai, China, conducted this study. It was supported by two grants from the Construct Program of the Key Discipline of State Administration of Traditional Chinese Medicine of the People's Republic of China and by the Science and Technology Development Fund of the Shanghai Municipal Public Health Bureau.

Deep vein thrombosis (DVT) is an extremely common medical problem that mainly affects the large veins in the lower limbs. It occurs either in isolation or as a complication of other diseases or procedures, especially surgical operations. In previous studies, the researchers had reported the protective effect of combined electrical stimulation at Taichong (LR 3), Zusanli (ST 36), and Sanyinjiao (SP 6) on postoperative DVT prevention in patients with gastrointestinal malignancies. ${ }^{\dagger}$ In the current study, the researchers investigated the DVT prevention effect of different acupoint combinations to detect the functions of each acupoint and plan appropriate nursing care for each individual patient based on differentiation of symptoms and signs.

One hundred and sixty elderly bedridden patients who underwent major surgeries between January 2010 and October 2011 agreed to participate. Patients were included if they met the following criteria: (1) age of > 60; (2) underwent major surgery; (3) operative duration of $>2$ hours; (4) in postoperative bed rest and could not get out of bed; and (5) signed an informed consent form. Subjects were excluded if they: (1) were noncompliant; (2) dropped out halfway during the study for various reasons; (3) had a condition that made it impossible to implement interventions, such as with lowerlimb infections; and (4) were in critical condition making it impossible to to implement interventions.

Subjects were randomized into 4 equal groups of 40 subjects each as follows:

(1) Conventional-care group-routine care + raising the lower extremities and postoperative symptomatic care

(2) Invigorating and promoting Qi group-routine care + bilateral transcutaneous electrical stimulation (TES) at Taichong (LR 3) and Zusanli (ST 36) in two 20-minute sessions per day for 1 week

(3) Blood-activating and Damp-eliminating grouproutine care + bilateral TES at Yinlingquan (SP 9) and Sanyinjiao (SP 6) in two 20-minute sessions per day for 1 week
(4) Acupoint-combination stimulation group-bilateral TES of a combination of four acupoints: LR 3, ST 36, SP 9, and SP 6 in two 20-minute sessions per day for 1 week.

Outcome measures included Severity of DVT and the chief complaints of the patients; Color Doppler flow imaging on the 7 th preoperative and postoperative days (diameter, blood-flow velocity, and thrombosis were examined in the external iliac veins, femoral veins, popliteal veins, and deep-calf veins; and coagulation function (D-dimer levels and blood viscosity were determined 7 days pre- and post operation).

Two patients in the conventional-care group were diagnosed with calf DVT. None of the patients in the other three groups developed DVT or leg swelling and pain.

Accelerated flow rates in the bilateral femoral veins, bilateral popliteal veins, bilateral deep-calf veins, and bilateral external iliac veins were observed in the invigorating and promoting Qi group $(p<0.05)$, the acupointcombination stimulation group $(p>0.05)$. Differences in diameter changes of the vessels, however, were not significant $(p>0.05)$.

The postoperative whole-blood viscosity and plasma viscosity in the conventional-care group were significantly higher than those in the other three groups, while differences among the other three groups were not statistically significant. Postoperative D-dimer levels in the conventional-care group were significantly elevated, compared with those in the other three groups; comparisons between the remaining three groups showed no statistically significant differences.

In the current study, the effect of electrical stimulation at different acupoints on preventing DVT was investigated. Combined stimulation at SP 9 and SP 6 or at LR 3 and ST 36 had an effect equal to that of combined stimulation at all four acupoints in terms of activating circulation and accelerating lower-limb blood flow. This may be a potentially safe and effective DVT-prevention method.

${ }^{\dagger}$ Hou LL, Yao LW, Niu QM, et al. Preventive effect of electrical acupoint stimulation on lower-limb thrombosis: A prospective study of elderly patients after malignant gastrointestinal tumor surgery. Cancer Nurse. 2013;36(2):139-144.

Address correspondence to: Wadie I. Najm, MD, MEd Department of Family Medicine \& Geriatrics \& Susan Samueli Center for Integrative Medicine and Executive Health University of California, Irvine 101 The City Drive, Building 200 \#835 Orange, CA 92868

E-mail: winajm@uci.edu 